\title{
ANÁLISIS DE PRÁCTICAS PERFORMATIVAS Y FARMACOPORNOGRÁFICAS EN LOS RELATOS AUTOBIOGRÁFICOS DE UN SUJETO/CUERPO EN MUTACIÓN
}

\author{
ANALYSIS OF PERFORMATIVE AND PHARMACOPORNOGRAPHIC PRACTICES \\ IN THE AUTOBIOGRAPHIC TALES OF A SUBJECT / BODY IN MUTATION
}

Nastassja Mancilla Ivaca Universidad Austral de Chile

\section{Resumen:}

Se analiza en el contexto de la sociedad heteronormativa chilena, desde los postulados de Judith Butler (2007) y Beatriz Preciado (2008), las prácticas performativas y farmacopornográficas en los relatos autobiográficos publicados a modo de diario de vida online de una mujer transexual. Se propone que, las prácticas analizadas son respuestas a procesos de subjetivación de un sujeto/cuerpo en mutación.

\section{Palabras clave:}

transexualidad; relatos autobiográficos; farmacopornografía; performatividad.

\section{Abstract:}

The performative and pharmacopornographic practices in the autobiographical stories published as a transsexual woman's online diary are analyzed in the context of Chilean heteronormative society, as taken from of postulates Judith Butler's (2007) and Beatriz Preciado's (2008). It is proposed that the practices analyzed are responses to processes of subjectivation from of a subject/body mutation.

\section{KeYWORDS:}

transsexuality; autobiographical stories; farmacopornography; performativity. 


\section{Presentación contextual}

En 1947, el psicólogo John Money acuña el término gender (género), que luego emplearía clínicamente para plantear la posibilidad de modificar el sexo de los bebés intersexuales (Preciado, 2008; Spargo, 2004), con la utilización de la tecnología clínica y hormonal. En 1953, el ex soldado norteamericano George W. Jorgensen se transforma en Christine, el primer transexual conocido mundialmente alsometerse a una cirugía de reasignación de sexo. En 1983, la transexualidad (o disforia de género) se incluye en la lista del DSM IV ${ }^{1}$ como patología mental, dejade serlo el 2013 en su quinta versión (Vicario, 2013), pero se mantiene como trastorno en la Clasificación Internacional de Enfermedades (CIE), de la OMS desde 1975 (Tena, 2013).

En Chile, Marcía Alejandra Torres Mostajo de Antofagasta se convierte en la primera mujer transexual en realizarse una cirugía de reasignación de sexo en 1973 (Rivera, 2012). La operación -de muchas más que vendrían- se realiza luego de meses de análisis de especialistas, pertenecientes a la entonces Sociedad Chilena de Sexología Antropológica $^{2}$ del Hospital Clínico de la Universidad de Chile. El diagnóstico fue un pseudo-hermafroditismo (González, 2007), que se identificó enlos rasgos de su cuerpo, producto de hormonas ingeridas que no declaró a losmédicos. Además, los informes psiquiátricos avalaron la prescripción.

El cambio de sexo, que planteaba la Sociedad de Sexología, era una reubicación a nivel biológico y social de los sujetos intersexuales, para: completar la definición de sexo (Quijada, 1968). Por ello, era necesario el tratamiento quirúrgico ymédico, y también, se debían considerar las dimensiones jurídicas, morales, antropológicas y psiquiátricas, para lograr la normalización de los individuos.

El cambio de identidad legal se puede realizar en Chile por medio de la Ley 17.344, promulgada durante el Gobierno de Eduardo Freí Montalva. Los requisitos para el cambio de nombre de un sujeto consideran: certificar legalmente el tratamiento y transformación bio-médica o quirúrgica (Gauché, 2013); la validación psiquiátrica para el procedimiento, y comprobar que se lleva una vida como hombreo mujer por al menos 5 años, para demostrar que su sexo y nombre no corresponden con la realidad. Existen 30 días legales durante los que una persona puede oponerseal cambio que solicita un individuo, por medio de argumentos que sean validos segúnla jurisprudencia.

1 Por sus siglas en inglés Diagnostic and Statistical Manual of Mental Disorders (Manual Diagnostico y Estadístico de los Trastornos Mentales).

2 Fundada en 1965. Desde 1988 se constituye como corporación de derecho al obtener su personalidad jurídica, y se conoce como Sociedad Chilena de Sexología y Educación Sexual.

3 Ley No. 17.344. Autoriza el cambio de nombres y apellidos en los casos que se indica. Modifica Ley № 4.808, sobre Registro Civil. Revisar: http://www.cambiodenombre.cl/ley.html
El proceso legal descrito, que se puede realizar actualmente en Chile ${ }^{4}$-a través de mucha burocracia y que ha favorecido a contados sujetos-, deja a criteriodel tribunal el cambio de sexo y de nombre a la vez. Tal como señala Foucault (2000), en sus cursos en el College de France entre 1974 y 1975 -publicados en el libro Los Anormales- lo que estaría en juego es la decisión de si el sujeto corresponde con lo normal. Lo que estaría dado por prácticas de instituciones que poseen en este caso, la autoridad del discurso médico/psiquiátrico y/o jurídico, que por medio de la técnica de la norma realizan la administración y control de la sexualidad de los sujetos (Foucault, 2000).

1.1. ReLATOS AUTOBIOGRÁfICOS: DE HOMBRE A MUJER TRANS

El presente trabajo pretende analizar el diario online escrito por Claudia Sayen Jacqueline Ancapan Quilape, quien describe a través de relatos de vida autobiográficos su paso de hombre a mujer, o Male to female (M2F) ${ }^{5}$ (Preciado, 2011). Los relatos son publicados desde el 13 de agosto de 2012 de manera periódicaen la página de facebook online: El diario vivir de una mujer transexual chilena, enla cual se narran experiencias de un sujeto/cuerpo en mutación (Preciado, 2008).

Siguiendo a Juan José Pujadas (2002) en relación a los relatos de vida, el tipo de documentos a analizar en este trabajo corresponderían al de tipo autobiográficos que no son motivados por el investigador (Pujadas, 2002). Cabe mencionar, que los documentos de vida como tales comienzan a presentar un gran interés de análisis a mediados del siglo XX por la sociología. Juan F. Marsal en su artículo “Historias de vida y Ciencias sociales", plantea que los relatos o documentos biográficos deben seranalizados considerando las condiciones históricas en que se escriben, lo que les otorga corpus metodológico y teórico propio (Sarabia, 1985).

Los relatos autobiográficos han sido utilizados por autores para introducir y analizar diferentes temáticas. Por ejemplo, Garfinkel (1967), en su obra Etnomethodology, utiliza la vida de Agnes, un transexual, para estudiar procesos de toma de roles y etiquetado; Bogdan (1974), estudia el paso del género masculino al femenino a partir de la vida de Jane Fry; y Foucault utiliza la vida de Pierre Riviere, un asesino francés

$4 \quad$ Actualmente en el país se discute la Ley de Identidad de Género, donde aún existen desacuerdos sobre cómo se puede llegar a una aplicación que no sea discriminatoria sobre los aspectos que establecen el cambio de identidad, ya que no es uno de los objetivos de la legislación solucionar esta problemática.

5 Como señala Preciado en el Manifiesto Contrasexual a pie de página: "las expresiones «F2M (female to male) y «M2F» (male to female) son fórmulas de autodenominación surgidas de la comunidad transexual anglosajona para nombrar, respectivamente, las personas en transición hormonal y/o quirúrgica hacia la masculinidad o la feminidad" (Preciado, 2011:22).

Revista Internacional de Culturas y Literaturas, abril 2015

ISSN: 1885-3625 
para analizar el discurso jurídico, y del hermafrodita Herculin Barbin o Alexina B. como parte de su genealogía de la sexualidad ${ }^{6}$.

\section{Tensionando lo heteronormativo}

La búsqueda del sexo verdadero, como plantearía Michel Foucault (1985) en la presentación del diario de vida del hermafrodita Herculin Barbin, o Alexina B., comenzaría a partir del siglo XVIII por medio de concepciones jurídicas y formas de control administrativo sobre el individuo en los Estados modernos, preocupación que nace desde la clase burguesa. El discurso médico así tendría la función de descifrar el sexo verdadero y establecerlo, y desde el derecho desaparecería la voluntad de elegir ${ }^{7}$, dando paso a la decisión del experto ante el "error" y las irregularidades de la sexualidad, "en un sentido filosófico más tradicional: una manera de proceder inadecuada a la realidad" (Foucault, 1985:14).

El análisis de Foucault sobre la Historia de la Sexualidad, publicado por primera vez en francés en 1976, plantea que la sexualidad se comienza a administrar, como mencionábamos anteriormente, a través de la técnica de la norma como dispositivo de control del poder/saber (Foucault, 2000; 2005), saliendo del ejercicio del poder disciplinar anterior al siglo XVIII. A partir de esto, señala la aparición de una tecnología del sexo, que identifica como dispositivo de la sexualidad y dispositivo de alianza, los cuales son discursos normativos, que funcionarían por medio de la normalización de prácticas que conciernen al sexo (Foucault, 2005). Este tipo de prácticas, serían normalizadoras/creadoras de sujetos y de sexualidad.

Según el autor, el dispositivo de alianza en este entramado de poder/saber estaría dado en el núcleo de la familia del siglo XVIII: por medio del matrimonio hombre/mujer, el parentesco, la trasmisión de bienes, y reproducción económica, querepercutirían en la instalación del discurso de una sexualidad normal/anormal y lo permitido/ prohibido. El dispositivo de sexualidad en cambio, "no tiene como razón de ser el hecho de reproducir, sino el de proliferar, innovar, anexar, inventar, penetrar los cuerpos de manera cada vez más detallada y controlar las poblaciones de manera cada vez más global" (Foucault, 2005:130). El dispositivo de alianza con sus restricciones y coerciones, sería productor de deseo y de sexualidad, permitiendo el funcionamiento

6 No aparece en la Historia de la Sexualidad (1976) específicamente, pero en la presentación del diario de vida de Herculin Barbin o Alexina B. retoma planteamientos de ese trabajo.

7 Como plantea el autor, en la edad media, en el caso de los hermafroditas en quienes se yuxtaponían dos sexos, el padrino o padre -que "nombran" al niño- en el momento del bautismo determinaban el sexo. "Pero más tarde en el umbral de la edad adulta, cuando se aproximaba el momento de casarse, el hermafrodita era libre de decidir por sí mismo si quería continuar siendo del sexo que se había le había atribuido o prefería otro. La única condición era la de no cambiar nunca más, y mantener hasta el fin de sus días lo que entonces había declarado, bajo pena de sodomía" (Foucault, 1985:12) del dispositivo de la sexualidad que amplía su campo de dominio y control, y sería la base del poder/saber de lo heterosexual.

La heterosexualidad es ante todo un concepto económico asentado en las relaciones de producción (Preciado, 2008), que se habría manifestado desde la antigüedad por el intercambio heterosexual y la distribución de mujeres (Wittig, 2006; Butler, 2007), que sería el medio para vincular a los hombres. La teórica sobre género, Monique Wittig, criticó desde el psicoanálisis a lo político y filosófico, al considerarlos como prácticas de los discursos de la heterosexualidad. En efecto, estos“nos oprimen en la medida en que nos niegan toda posibilidad de hablar si no es en sus propios términos y todo aquello que los pone en cuestión es enseguida considerado como "primario»" (Wittig 2006:49).

La autora propone que esto es producto de un pensamiento heterosexual que plantea la distinción entre naturaleza/cultura, basado en una serie de conceptos primitivos y de relaciones totalizadoras que abarcarían lo social, lo político, locultural, lo histórico, las subjetividades, lo real, etc.:

\begin{abstract}
Y por mucho que se haya admitido en estos últimos años que no hay naturaleza, que todo es cultura, sigue habiendo en el seno de esta cultura un núcleo de naturaleza que resiste al examen, una relación excluida de lo social en el análisis y que reviste que caste al exame un uctabilidad en la cultura como en la naturaleza: es la relación heterosexual. (Wittig, 2006:51)
\end{abstract}

\subsection{TECNOGÉNERO}

La técnica de la norma adelantada por Foucault, plantea que en estas relaciones de dominio lo que se intenta administrar es la no correspondencia con lo real o normal, y sería la base de la normalización de discursos y prácticas de corte heterosexual, por medio de procesos de subjetivación que actúan sobre los sujetos, y el control de estos por medio del dispositivo de la sexualidad. En ese sentido,estaríamos ante un régimen heteropatriarcal, o heteronormativo ${ }^{8}$, donde las subjetividades contrarias, estarían practicando una especie de terrorismo de género (Preciado, 2008), el cual se debe controlar.

La noción de género en este régimen heteronormativo, desde el trabajo de Teresa de Lauretis, plantea el género como producto y constructo de tecnologías sociales, como el cine, los discursos epistemológicos, de las teorías, y aquellos que provienen desde fuera del contrato social heterosexual a nivel micropolítico (De Lauretis, 2000). Este diversificado entramado de aparatos tecno-sociales y también, bio-médicos, dice

8 Término que aparece por primera vez en francés según Monique Wittig 2006).

Revista Internacional de Culturas y Literaturas, abril 2015 
la autora, amplían la visión de la tecnología del sexo de Foucault, alas que se somete a los sujetos/cuerpos, que denomina tecnogénero.

Los postulados que situaban el género como diferencia sexual y del lenguaje son superados en la conceptualización de De Lauretis, para plantear un tecnogénero que debe ser analizado desde una semiótica política. En ese sentido, Beatriz Preciado (2008) plantea que se debe mirar también, lo semiótico técnico producto del tecnocapitalismo avanzado. “Digámoslo cuanto antes: este nuevo modelo no se caracteriza simplemente por la transformación del sexo en gestión política de la vida, sino, y sobre todo, por el hecho de que esta gestión se opera a través de las nuevas dinámicas del tecno-capitalismo avanzado" (Preciado, 2008:81). Por lo cual, lo heterosexual hombre/mujer, en el contexto actual comienza a tensionarse e incluso a desaparecer como "naturaleza" ante la técnica.

\section{Prácticas Performativas y farmacopornográficas}

Las propuestas de Tamsin Spargo (2004) sitúan lo heteronormativo como dependiente de la homosexualidad, y productor de ésta al someterla a la sujeción de la norma, siguiendo los análisis de Foucault. Para los fines de este trabajo, se plantea que esta relación productora también se aplica para la transexualidad al abordar la problemática desde el campo de la inteligibilidad teórica. La autora identifica a partir de las ideas del filósofo francés que el sexo es una categoría ficticia, y donde el cuerpo no es "naturalmente", como se podría pensar, "sexuado", sino más bien, esto se produce a través de procesos culturales.

El filósofo, planteó que el control de la sexualidad es inmanente al cuerpo social y no una relación de dominador/dominado (Foucault, 2005). En los trabajos deJudith Butler (2007), esta inmanencia la podríamos ver en lo performativo; y en la atención que hace Beatriz Preciado (2011) en los regímenes semióticos políticos y técnicos, que analiza desde la farmacopornografía (Preciado, 2008; 2011).

\subsection{Performatividad}

El análisis basado en las prácticas discursivas (Foucault, 2009) y lo performativo que plantea Butler (2007) presentes en el diario de Claudia Ancapan, sejustifica en que, como advierte Teresa De Lauretis (1992), esto respondería a un tipode confrontación que sería "discursiva por naturaleza -en el sentido de que el lenguaje y las metáforas están siempre en la práctica, en la vida real, donde reside en última instancia el significado" (De Lauretis, 1992:13). Considerando que el cuerpo como fenómeno social es vulnerable y está expuesto a los demás (Butler, 2010), existen respuestas propias del sujeto a determinadas situaciones que se generan de lasprácticas y dispositivos, y que podríamos situar como la respuesta a esteenfrentamiento del cuerpo ante procesos de subjetivación

Este «se enfrenta a» es una modalidad que define al cuerpo. Y, sin embargo, esta alteridad obstrusiva con la que se topa el cuerpo puede ser, y a menudo es, lo que anima la capacidadde respuesta a ese mundo. Esta capacidad puede incluir una amplia gama de afectos, como placer, rabia, sufrimiento o esperanza, por nombrar sólo unos pocos. (Butler, 2010:58)

Para Butler (2007), el cuerpo es una práctica significante, que es lo que denomina como performativo, y que estaría dado por la repetición de significados que se legitimarían a través de la ritualización de ciertas prácticas corporales creando el efecto de género. En este punto, la noción de género y la experiencia discursiva está determinada por los limites del discurso hegemónico que responde a lo binario, “De esta forma, se elabora la restricción dentro de lo que ese lenguaje establececomo el campo imaginable del género" (Butler, 2007:59). En el caso del diario de Claudia, vemos como se describen este tipo de restricciones de género y a la vez, la capacidad de respuesta en sus relatos ante determinadas situaciones dominadas por eldiscurso y las fronteras de lo heteronormativo, producto de procesos de subjetivación.

3.2. Farmacopornográfico

El análisis plantea así que la capacidad de respuesta se manifiesta a través de cuerpos que generan discursos, y es lo que Preciado (2011) denomina cuerpos hablantes que reconocen a otros como cuerpos hablantes. Donde existen prácticas discursivas y performativas, que producen inteligibilidad (Preciado, 2008). Siguiendolas propuestas de la autora, nos encontraríamos ante un campo práctico de saber, donde aparece la noción de tecnogénero que toma desde Teresa de Lauretis, y que sería la forma de analizar la sexualidad, el género y el cuerpo, en lo que denomina farmacopornografía.

Lo farmacopornográfico según Beatriz Preciado (2008), surge en el contexto global y mediático por "procesos de gobierno biomolecular (fármaco-) y semiótico técnico (-porno) de la subjetividad sexual" (Preciado, 2008:32). La preocupación de la autora, se basa en la autoridad otorgada a la ciencia, o más bien tecnociencia, en la modernidad donde ésta ya no sólo describe la realidad, sino también tiene la autoridad de crearla. El cuerpo surge así como artefacto naturalizado, y las subjetividades de estos cuerpos/ sujetos aparecen definidos por sustancias que dominan sus metabolismos, de forma toxipornográfica. Que sería la nueva forma de gestión política de la vida por las nuevas relaciones de producción del tecno- capitalismo. Así, se revisan los relatos que se presentan a continuación, identificandolas prácticas farmacopornográficas que realiza el sujeto en el contexto heteronormativo de la sociedad chilena. 


\section{Sujeto/CUerpo en mUtación: ANÁLISIS}

Los relatos autobiográficos de Jacqueline Ancapan analizados para los objetivos de este trabajo, fueron un total de 6 escritos durante el año 2012 y 2013. Para su selección se utilizó como criterio de análisis las propuestas teóricas sobre performatividad y farmacopornografía que daban cuenta de un sujeto/cuerpo en mutación que se enfrenta a procesos de subjetivación de su sexualidad. Juan Carlos, como era conocida anteriormente la mujer transexual que escribe los relatos, comenzó su paso de male to female cuando estudiaba la carrera de Obstetricia en la Universidad Austral de Chile, en Valdivia. Donde realiza sus primeras experimentaciones con hormonas para comenzar la transformación de su cuerpo, al conocer sobre este tipo de tratamientos en libros de medicina disponibles en la biblioteca de la institución donde cursaba sus estudios:

Ya en primer año de universidad comenzado a tomar hormonas y graduarlas en mí, ver en 3 meses sus resultados, me tornada muy sensible y ver cómo crecían mis senitos era como nacer de nuevo, sólo mis amistades más cercanas sabían de mi felicidad y avances, mis profesoras y compañeros de universidad sólo rumoreaban yo siempre con terror a que

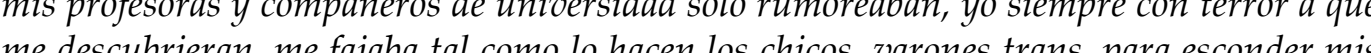
me descubrieran, me fajaba tal como lo hacen los chicos varones trans, para esconder mis senos. $(14 / 06 / 2013)$

Las prácticas farmacoporgráficas que realiza Claudia van desde la utilización de hormonas hasta su interés por realizarse una cirugía de reasignación de sexo. Anteesto, incluso llega a automutilar su pene por su deseo de tener, como ella misma señala, una "vagina funcional", efecto del exceso de poder del discurso heteronormativo. Y es en este punto donde podemos observar también la dimensión performativa que emana desde el discurso de ciertas instituciones autorizadas; en consecuencia, en las prácticas que son inmanentes a los sujetos producto del contrato heterosexual.

Y en una noche de locura, después de haber sido despreciada por ser trans por un hombre enValdivia a quién amé muchísimo, llorando en mi cama, quería castrarme y una cicatriz penosa queda de ese momento cuando yo pensaba que no podía acceder al amor tan sólo por no tener genitales femeninos (...) Los libros de psiquiatría se llenan la boca con nuestras vidas, pero no dicen el sufrimiento, los desamores, las increíbles proezas para generar el dinero, aun no teniendo DNI o cédula de identidad acorde, para asic hace con nuestras vidas. (14/06/2013)

En los relatos de Claudia, se pueden identificar las diversas instituciones que funcionan en el contexto chileno, como son las iglesias, la medicina, la psiquiatría, las instituciones de educación, entre muchas otras. En consecuencia, lo trans aparece como una oportunidad para cuestionar y analizar prácticas que parecieran estar naturalizadas en los sujetos, y que se describe en los documentos analizados a modo de reflexión de las experiencias vividas:
Ciertamente he llegado al punto de pensar en que sin los varones hetero normados yo jamás habría tomado conciencia de que soy una mujer trans. Y lo digo por aquellas preguntas constantes de intriga y ganas de satisfacer curiosidades innatas a todo lo desconocido que representamos las mujeres trans y que escuché y viví durante mis años de construcción trans. Siempre me quedé pensando en cómo responder preguntas tales como ¿Qué es set travesti o rransexul Clau travesti o thansexhal, Claudia?, ¿Uds. tienen sexo como las mujeres, cierto? ¿Esos senos, ¿Por qué siempre están en las calles prostituyéndose? (21/08/2013)

La coerción de lo performativo que Preciado (2008) señala desde Butler, se manifiesta en las relaciones que se establecen entre los sujetos/cuerpos hablantes, ante ciertas situaciones que dan cuenta de relaciones de poder que muchas veces pasan desapercibidas ante las descuidadas conciencias que incurren en ellas. Y que desembocan y generan procesos de subjetivación. Como señala Butler (2010), el género se genera ante determinados contextos, que bien se identifica en los relatos deClaudia al comentar que el discurso heterosexual dio cuenta sobre su condición de mujer trans.

Los discursos autorizados se avalan en prácticas que responden a lo performativo y farmacopornográfico, y que incluso aparecen como inmanentes al cuerpo social, como medio para justificar la correspondencia del sujeto/cuerpo con lo real -entendido en conceptos binarios- que se establecen para la validación ante la norma y a la vez como proceso de creación de realidad.

\section{Hoy muchas trans tienen que vivir este calvario porque para la ciencia es válido tener la certeza de que "esta niñita se puede arrepentir de cortarse los coquitos, tenemos que asegurarnos". iQué martirio! La primera psicóloga que me vio, pensó que yo era una mujer que quería ser un hombre y durante todo su interrogatorio, me decía que tenía que construir mi identidad paso a paso, yo le decía sabe me visto de mujer desde los 5 años, séque tengo que caminar a la transición pero usted está equivocada yo nací hombre genitalmente hablando y soy mujer de razón y sentimiento y así deseo seguir viviendo. (26/04/2013)}

En un fragmento de los relatos analizados se narra cómo ciertos sucesos, en los cuales Claudia interactuó con otros individuos, fueron detonantes de una necesidad de adecuación, ya que los espacios y las mentes heteronormadas delimitan el encuentro con la alteridad:

...cuando se filtraron mis fotos de Claudia aquella mujer (yo misma) que disfrutaba cada instante en Valdivia (otra ciudad de Chile) en mi época universitaria, aunque en las fotografías era una mujer tal vez algo sobrecargada, me parecía insólito todo lo que se hablaba de mí: que siempre fui una trabajadora sexual y transformista y gay, que el vih recorría mis venas por mi estilo de vida, que era drogadicta y que no tenía moral para ser matrona, (a modo de burlas) era lo más suave que escuchaba, tal vez por ello, cuando llegaba a un lugar a trabajar, no duraba mucho, siempre estaba en constante movimiento laboral buscando trabajo en otro lugar... (09/12/2012)

Las prácticas performativas están presentes en la vida real desde que empezamos a experimentar procesos de subjetivación a temprana edad. Basta constatar que al nacer somos asignados como hombre o mujer. Y muchos de los relatos que aquí se han presentado son situaciones que hemos experimentado, con la excepción de que 
cada cual lo ha vivido desde diferentes lugares del tablero de juego,unos como meros espectadores y otros como protagonistas / víctimas y/o victimarios a través de ciertas prácticas discursivas:

Los niños para darme la bienvenida a la escuela me invitaron a jugar, la profesora dijo unas frases que me marcaron "los niños Juan Carlos no juegan a la ronda, ni con muñecas, usted debe estar con los niños jugando futbol se va inmediatamente con ellos". (16/05/2013)

\section{REFLEXIONES FINALES}

La transexualidad como campo de poder/saber entrega elementos sustanciales para analizar la sociedad heteronormativa en sus diferentes dimensiones en Chile, considerando que el país aún se caracteriza por mantener una mirada conservadora instalada en las instituciones oficiales, en las legislaciones, en el discurso médico, laboral, educacional y socio-cultural, que hacen invisibles a la alteridad y diferencia de los individuos. Sin embargo, las realidades que nos muestran los sujetos/cuerpos dan cuenta de que a pesar de esta concepción aún muy patriarcal, que incurre en violentos procesos de subjetivación, surge la oportunidad de reformular los discursos, y redefinir el contexto y sus significados, en el intento de no aceptarlos, o asumirlos como si pertenecieran a alguien omnipresente.

La necesidad de superar y tensionar lo heteronormativo, vendría a proponerse como resistencia ante el régimen capitalista impuesto a nivel biopolítico, por efecto de la capitalización de la vida y los cuerpos cada vez más en auge como mecanismo de mercado. Baste constatar y revisar los análisis de Beatriz Preciado para dar cuenta de esta situación. Por otro lado, en Chile y en los contextos latinoamericanos este tipo de prácticas son una dimensión a abordar y a proponer en el campo teórico que indaga en temáticas de sexo y cuerpo. Por ello, se propone que lo farmacoporgráfico y performativo como creador de realidades y, analizar el efecto de sus prácticas en los sujetos/cuerpos, puede ser un desafío al campo de saber/poder de la heterosexualidad. Además, suponen asumir que estamos ante la extinción y re- formulación de ciertas concepciones que de lo heterenormativo se emanan.

\section{REFERENCIAS BIBLIOGRÁFICAS}

Butler, J. El género en disputa. España, Barcelona, Ediciones Paidós Ibérica S.A. 2007. Butler. J. Marcos de guerra. México D.F., Editorial Paidós Mexicana S.A., 2010.

De Lauretis, T. Diferencias. Etapas de un camino a través del feminismo. Cuadernos Inacabados. Madrid, Horas y horas, 2000, pp. 33-70.

De Lauretis, T. Alicia Ya No. Madrid, Ediciones Cátedra, S.A., 1992. Foucault, M. Herculin Barbin llamada Alexina B. Madrid, Editorial Revolución, 1985.
Foucault, M. La Historia de la Sexualidad I. La voluntad del saber. Argentina, Siglo Veintiuno, 2005, pp. 93-160

Foucault, M. Los anormales. Buenos Aires, Fondo de la Cultura Económica, 2000.

Gauché, X. "Algunas reflexiones sobre las razones que justifican una ley de identidad de género para Chile y una mirada al proyecto de ley ingresado el 7 de mayo de 2013 al Congreso Nacional". Género, sexualidades y derechos humanos. Revista Electrónica Semestral del Programa Mujeres, Género y Derechos Humanos, Vol. 1, nº 2 (2013), pp. 49-59.

González, J. "Marcia Alejandra Torres Mostajo. La primera mujer transexual de Chile”. Entrevista en Profundidad. Biblioteca Digital INDH, 2007. Internet. 28- 08-13. http:// bibliotecadigital.indh.cl/handle/123456789/423

Preciado, B. Testo Yonqui. Madrid, Editorial Espasa, 2008.

Preciado, B. 2011. Manifiesto Contrasexual. Barcelona, Anagrama, 2011.

Pujadas. J.J. "El método biográfico: El uso de las historias de vida en ciencias sociales". Colección cuadernos metodológicos, $\mathrm{n}^{\circ} 5$. Madrid, Centro de Investigaciones Sociológicas, 2002, pp. 7-40.

Quijada, 0. Cambio de sexo. Puntos de vista antropológico, biológico, embriológico, genético, clínico endocrinológico, psiquiátrico, religioso católico y jurídico: con un apéndice sobre correcciones quirúrgicas. Buenos Aires, Almendros,Joaquín (Edit.), 1968, pp. 9-29.

Rivera, A. "Informe sobre Chile -Violación a los DDHH de PersonasTransexuales Quinta Ronda del Examen Periódico Universal ONU (Organización de las Naciones Unidas)". Biblioteca digital INDH, 2012. Internet. 10-01-14.http://bibliotecadigital.indh.cl/bitstream/ handle/123456789/172/EPU- OTD.pdf? sequence $=1$

Sarabia, B. "Historias de vida". Revista Española de Investigaciones Sociológicas, 29 (1985), pp. 165-186.

Spargo, T. Foucault y la teoría queer. Editorial Gedisa, 2004, pp. 53-72.

Tena, F. "Sacudirse la tutela médica. Hacía la despatologización de latransexualidad". Revista Andaluza de Antropología, 5, (2013), pp. 35-65.

Vicario, M. H. "Transexualidad en pediatría”. Revista Pediatría Integral,(2013), pp. 13 15. Internet. 09-01-14. http://www.sepeap.org/secciones/documentos/pdf/1315\%20SEM\%20Hidalgo.pdf Wittig, M. El pensamiento heterosexual y otros ensayos. Barcelona, Editorial EGALES, 2006. 